\title{
Lifting frontal apoyado en malla de polipropileno
}

\author{
Brow lift supported on \\ polypropylen mesh
}

\author{
Aracil Kessler, J .P.*, De Francia Cachon, S.**, Vicent Dols, A.***, Llorens Salvador, M.***, \\ Esteban Masanet, M.*****, Martin García, R.F.***, Llobet Merce, J .***
}

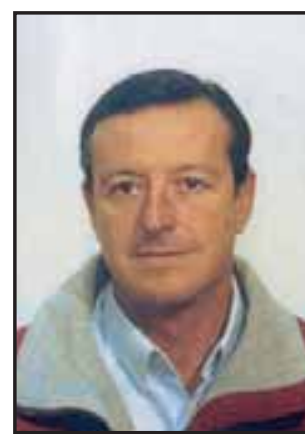

Aracil Kessler, J.P.

\section{Resumen}

La región frontorbitaria tiene una especial significación en el contexto del envejecimiento facial. La literatura cuenta con numerosa bibliografía que trata sobre este problema. Se considera como una unidad diagnóstico-terapéutica en los últimos tiempos y la evolución de la cirugía hace que se vaya hacia soluciones cada vez más fisiológicas y mínimamente invasivas. Nuestro trabajo queremos que sea un paso más en esa dirección.

Sobre una muestra de 25 pacientes en los que se trató la región frontorbitaraia con diferentes procedimientos, y que además mostraban un descenso de la altura de las cejas, se presenta una técnica de suspensión frontorbitaria con mínima incisión, apoyada en una malla de polipropileno anclada en el músculo frontal y galea en el extremo inferior y en el periostio y la galea en su extremo superior en el plano supraperióstico y sin resección cutánea. Se emplea un sistema de medición de resultados basado en puntos del esqueleto facial

Los resultados han sido satisfactorios subjetiva y objetivamente, y comparables a los de otras técnicas más complejas y de mayor coste económico. Las complicaciones desarrolladas han sido leves y pasajeras.

Se valora porqué se decidió utilizar la malla de polipropileno, así como porqué se eligió un gramaje concreto. También se analizan las características que aportan el material y su uso en esta técnica como comportamiento ante la infección, tolerancia tisular, retracción cicatricial y adhesividad. Asímismo se justifica el plano de disección y colocación elegido, y se valora el sistema de medición para el control objetivo de los resultados.

La aportación de este trabajo consiste en desarrollar una alternativa dentro de las técnicas mínimamente invasivas para le reposición de la región frontorbitaria, sin resección de piel y con buenos resultados en la corrección de las arrugas frontales, comparables a los de otras técnicas de mayor complejidad y elevado coste económico.

\section{Palabras clave Ritidectomía frontal. Rejuvenecimiento facial. Malla quirúrgica. \\ Código numérico 260-1586}

The fronto-orbital region is of particular significance in the context of facial ageing. There is a considerable amount of research on the nature of this problem. This area has recently come to be considered as a therapeutic-diagnostic unit and surgical developments mean that focus is switching more towards less invasive physiological solutions. Our study aims to take another step in this direction.

Taking a sample of 25 patients, all of them undergoing different procedures on the fronto-orbital region and with a lowering of the eyebrows, a suspension technique was introduced with minimum incisions supported by a polypropylene mesh attached to the frontal muscle and galea in the inferior extreme and to the periosteum and galea in the superior extreme in the supra-periosteum plane and without skin removal. A measuring system based on points of the facial skeleton was used.

The results were subjectively and objectively satisfactory compared to those from more complicated and expensive techniques. Complications were of a slight and temporary nature.

This section carries out an assessment of the reasons for selecting a polypropylene mesh and specific grammage. In addition, the properties of the material are analysed and its use in this context of preventing infection, tissue tolerance, scar reduction and adhesiveness. Justification of the site of dissection and the choice of positioning, and an assessment of the course of medication given in order to produce a set of controlled and objective results, will also be carried out

The aim of this work is to develop an alternative within minimally invasive techniques for the restructuring of the fronto-orbital region without skin removal and with good results in the correction of facial wrinkles compared to other more complicated and expensive techniques.

Key words Brow lift. Facial rejuvenation. Surgical mesh.

Código numérico 260-1586

* J efe de Unidad de Cirugía sin Ingreso.

* Residente de Cirugía Plástica del Hospital «La Fe» de Valencia.

* Adjunto de Unidad de Cirugía sin Ingreso.

* Adjunto del Servicio de Oftalmología.

Unidad de Cirugía sin Ingreso. Consorcio hospitalario provincial de Castellón. Castellón (España). 


\section{Introducción}

Hinderer escribe en 1990 que "la imagen física envejecida, no se corresponde en muchas ocasiones con el deterioro de las capacidades físicas; no teniendo éste una relación directa con el sentimiento de juventud interior" (1).

Los estigmas del proceso involutivo propios del envejecimiento facial han sido motivo de múltiples estudios descriptivos y ensayos terapéuticos que han venido ocupando espacios importantes en la literatura de la especialidad (2). De la región fronto-orbitaria dice Caldeira (1996) que "tiene una especial significación en el contexto del envejecimiento facial, pues es la ventana de la expresión fisiognomónica que a veces con su aspecto de fatiga y de tristeza no refleja la realidad de una cara aún joven" (3).

Y de ello, la distopia ( y en concreto la hipotopia) de la cola de las cejas, que parece un detalle nimio, pero es el responsable de gran parte de los cambios del tercio superior de la cara que acompañan a los procesos propios del paso del tiempo. Esta hipotopia se produce por el proceso de reabsorción ósea frontal y por la ausencia de fijaciones profundas de la piel de la zona, con un descenso gravitacional secundario de los tejidos blandos sobre la fascia temporal y de la piel sobre el tejido fibrograso subciliar y preseptal (4).

La dermatocalasis del párpado superior, que es consecutiva a un envejecimiento cutáneo, no sólo se manifiesta a nivel del párpado, sino también a nivel de la frente. A menudo es la caída o descenso de la ceja lo que precipita un remanente cutáneo sobre el párpado superior. Si se interviniera sólo sobre éste, se reduciría, retirando una cantidad considerable de piel, la distancia que separa las cejas de las pestañas, lo que podría llevar a serias complicaciones (5).

La importancia de este hecho se refleja en el trabajo de Pollock y Gossman, que en un estudio prospectivo realizado en pacientes sometidas a blefaroplastia, resumen los posibles errores de juicio preoperatorio y los malos resultados postoperatorios consecuentes, indicando como primera causa la apariencia de pesadez lateral de la ceja (6). Por ello, desde la época de las grandes ritidectomías que culminaron a nuestro juicio con la técnica del "mask lift" de Tessier (7), la evolución ha llevado hasta los refinamientos actuales mediante los cuales se trata de corregir este problema con métodos endoscópicos u otros métodos de suspensión minimamente invasivos, como es la utilización de hilos arponados o a adminículos que anclan el complejo dermo-graso-aponeurótico a la calota o nuestra propia propuesta

Presentamos nuestra alternativa como un método de suspensión frontal lateral mínimamente invasivo, de gran simplicidad técnica, de muy buenos resultados y bajo coste tanto económico como de secuelas.

\section{Material y método}

Se ha ensayado el procedimiento en 25 pacientes, 18 mujeres y 7 varones con una media de edad de 53 años y un rango de entre 42 y 67 años.

Se realizó en todos los casos una evaluación previa de la totalidad de la región. Como criterio de selección se tomó la altura de las cejas en relación al borde ciliar, de modo que se aceptó a todos aquellos varones en los que al menos la mitad externa de la ceja estuviera por debajo del borde ciliar y a todas aquellas mujeres en las que al menos esa porción de la ceja quedara sobre dicho arco.

Se realizaron mediciones, en la fase preoperatoria y periódicamente en el postoperatorio, de una distancia sobre una línea perpendicular a la línea de Francfort y tangente al borde anterior de la apófisis zigomática del frontal (Fig 1). La distancia medida es la existente desde el borde piloso inferior de la ceja a la línea de Francfort.

A todos los pacientes menos a tres, se les realizó como complemento una blefaroplastia superior, y en los casos necesarios se realizaron otros refinamientos como tratamiento de la grasa subciliar, del prolapso

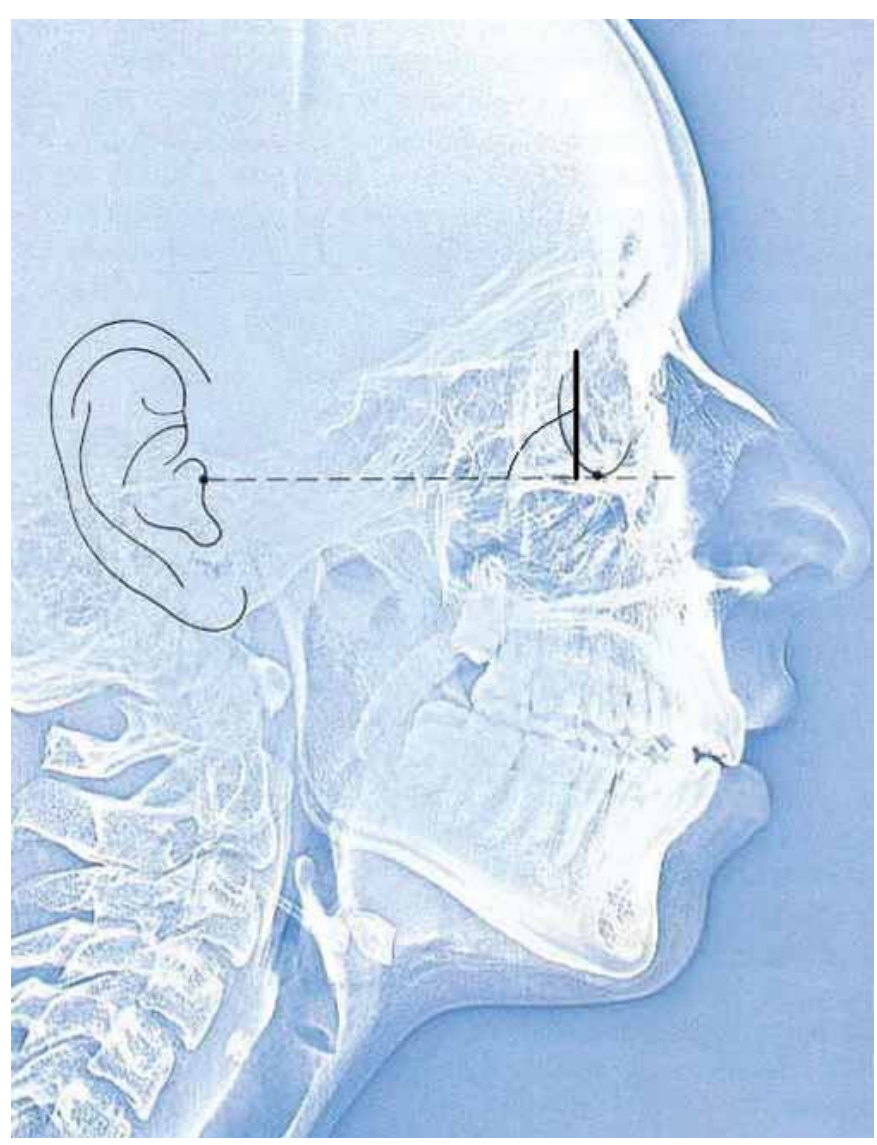

Figura 1: Perpendicular a la línea de Francfort, en trazo grueso, se expone la tangente anterior a la apófisis zigomática del frontal donde hacemos las mediciones. 


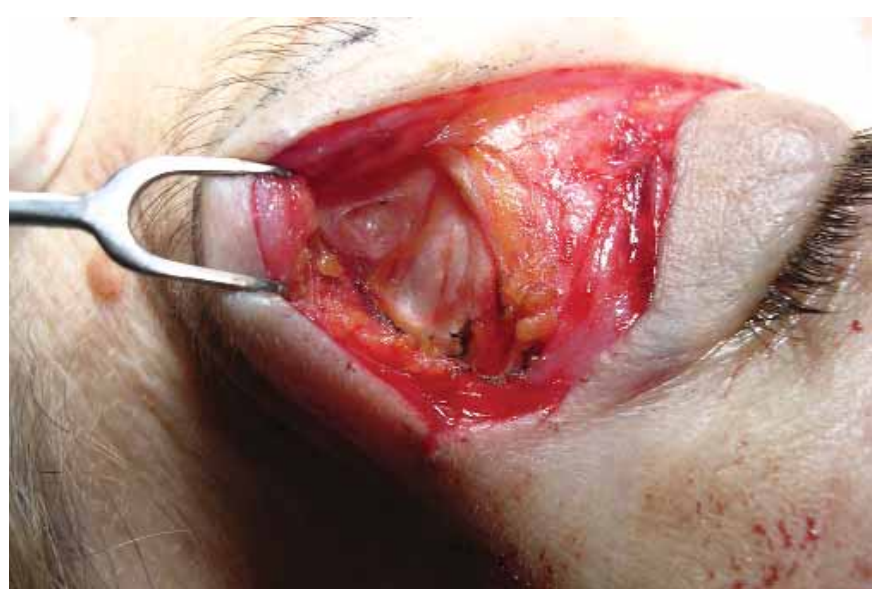

Figura 2 : La incisión inferior llega al plano perióstico. Nótese que se ha retirado el músculo orbicular preseptal.

de la glándula lagrimal, rebaje del borde orbitario, o tratamiento de los músculos depresores mediales (corrugador, procerus, etc).

Para realizar la suspensión, preparamos una porción de malla de una pieza de polipropileno monofilamentoso y de poro grande tipo I de la clasificación AMID (Tabla I) $(8,9)$, concretamente malla Hermesh $^{\circledast} 5$ flat mesh, que tiene un grosor de filamento de $180 \mathrm{~mm}$ y un diámetro de poro de $748 \mathrm{~mm}$, lo que representa un gramaje o densidad de $107 \mathrm{gr} / \mathrm{m}^{2}$.

\section{Técnica quirúrgica:}

Realizamos estudio preoperatorio rutinario y preparación previa de los pacientes con profilaxis antibiótica según protocolo con Eritromicina 500-1000 mg. parenteral en dosis única 2 horas antes del comienzo de la intervención y sedación con $2 \mathrm{mg}$. de Midazolam I.V. antes de comenzar el procedimiento. Rara vez se necesita reforzar con Propofol.

Se marcan las incisiones superior e inferior. La superior de $1 \mathrm{~cm}$. aproximadamente, horizontal, aprovechando alguna arruga frontal, cerca de la raíz del cabello o a su nivel según la altura de implantación, y un través de dedo por dentro del extremo lateral de la ceja (a la altura de la cresta temporal). También marcamos la situación teórica del nervio supraorbitario a $2.7 \mathrm{~cm}$. de la línea media.

La incisión inferior se marca en el borde piloso inferior de la ceja en su tercio externo o hasta la mitad externa, o se puede aprovechar la incisión de blefaroplastia superior, si se debe realizar ésta, ya que es bastante constante la asociación de este problema a la blefarocalasis superior.

Tras ello se infiltra con anestésico local: en nuestro caso lo hacemos con la misma mezcla que usamos para la blefaroplastia: Mepivacaina al 1 o al $2 \%$ con adrenalina a 1:200.000. Generalmente bastan 4 o $5 \mathrm{cc}$ para infiltrar la zona.

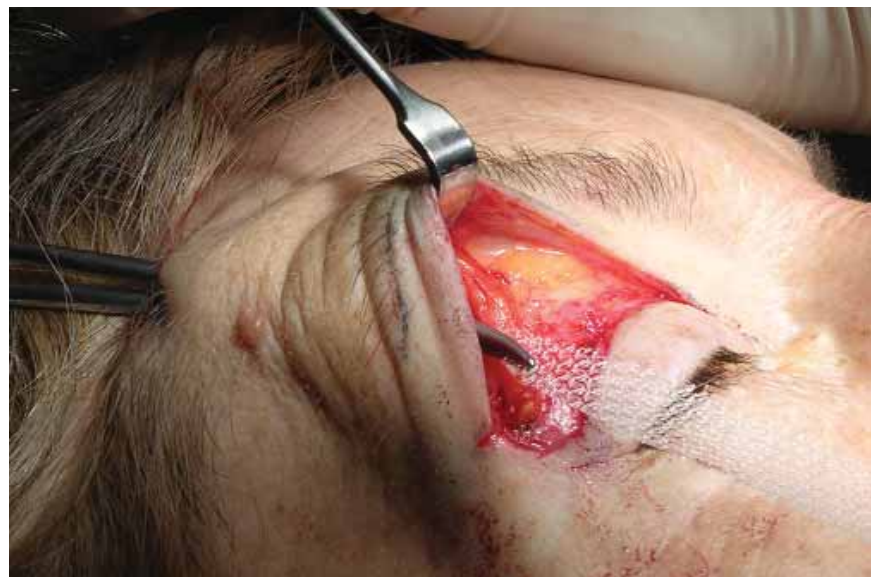

Figura 3 : Tunelización supraperióstica entre ambas incisiones y colocación de la malla

La incisión superior se realiza hasta llegar al periostio craneal, dejando la galea incidida y adherida al colgajo formado por la piel y el tejido celular subcutáneo. La incisión inferior, sea de blefaroplastia o ciliar, debe atravesar las fibras superiores del músculo orbicular del párpado, separándolas de las del frontal o la galea en el extremo lateral, o eliminando la porción preseptal del músculo orbicular como hacemos en las blefaroplastias superiores (Fig. 2), ya que parece consensuado de forma general el hecho, de que este músculo es el verdadero depresor del sistema frontorbitario $(3,10)$ y llegamos así a nivel del periostio ciliar, siempre externos al nervio supraorbitario para evitar lesionarlo. Retiramos asímismo el exceso de la bolsa grasa subciliar, corregimos el prolapso de la glándula lagrimal o rebajamos el reborde orbitario en los casos en que sea necesario. También es el momento de tratar, si fuera menester, los músculos depresores mediales (corrugador, procerus, etc.). En este momento se tuneliza por encima del periostio comunicando las dos incisiones.

Previamente hemos preparado una porción de malla de una longitud 2 ó $3 \mathrm{~cm}$. mayor que la distancia del reborde ciliar a la incisión superior (Fig. 3). El extremo inferior es de una longitud semejante a la porción de ceja que pensamos corregir, y el extremo superior igual o ligeramente mayor a la incisión superior. Pasamos la malla a través del túnel y fijamos el extremo inferior de la misma con dos o tres puntos de monofilamento de 5/0 o $6 / 0$ al resto de orbicular que se había quedado en el labio superior de la herida inferior y al arranque del músculo frontal y la galea que se confunden con el propio músculo orbicular (Fig 4).

Tirando de la malla desde su extremo superior, que salía por aquella incisión, se ensaya la corrección deseada y se fija con un punto transfixiante desde el labio superior de la herida superior (de $3 / 0$ o 4/0) cor- 


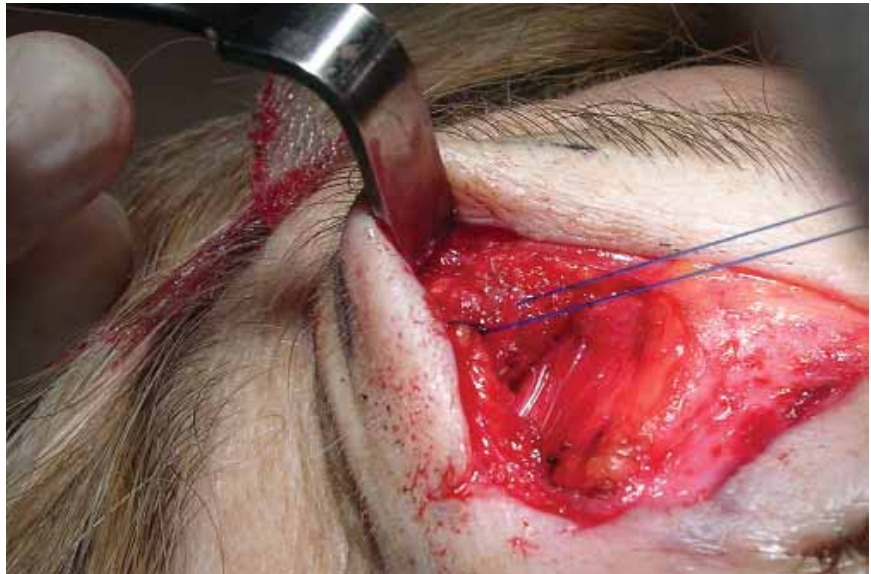

Figura 4 : La malla se ha sujetado con puntos sueltos a la galea a nivel de la incisión inferior

tando el exceso de malla y acomodándola por debajo de la galea (fig 5). Este punto nos sirve para fijar la malla a la galea en su punto, y así descargar la tensión durante la primera semana, en tanto que comienza la cicatrización y las fibras de colágeno y el resto de la trama comienzan a atravesar y fijar la malla.

Se fija la malla también a la galea a la altura de la incisión superior con uno o dos puntos de monofilamento 5/0 o 6/0 o incluso al periostio inferior. Tras ello, se cierra la piel de ambas heridas (nosotros lo hacemos con el mismo monofilamento).

\section{Resultados}

Podemos decir que los resultados han sido buenos si tomamos como base la encuesta de satisfacción hecha a los pacientes en que manifestaron que los resultados estaban a la altura de sus expectativas. Además hemos observado, y así lo han manifestado también los pacientes, la corrección o incluso desaparición de gran parte de las arrugas frontales horizontales (figura 6-11) .

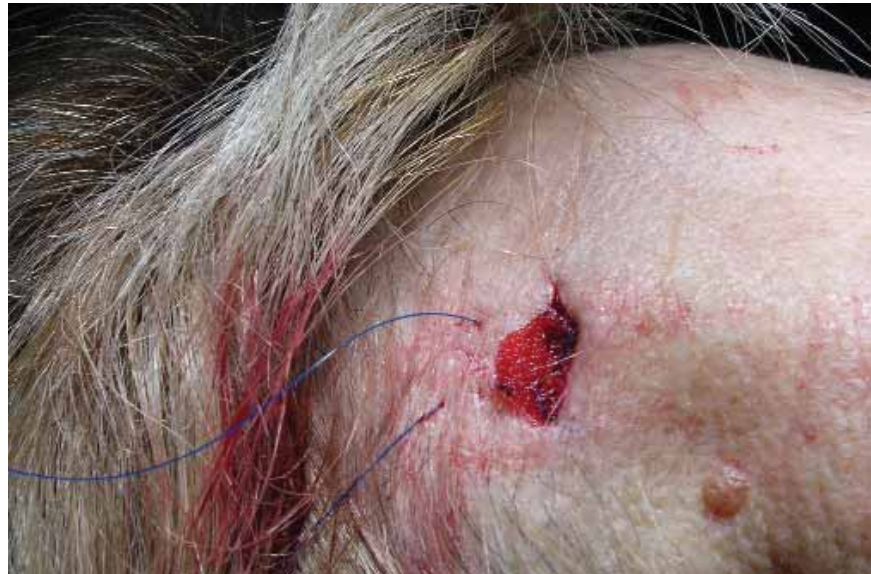

Figura 5 : La malla se sujeta a la galea a nivel de la incisión superior con puntos transfixiantes, eliminándose el exceso de la misma para acomodarla posteriormente en su plano

Si tomamos como base datos objetivos, los resultados de las mediciones de la línea Cilio-Francfort han resultado ser $0.9 \mathrm{~cm}$. más altos como media en las mediciones postoperatorias a los 6 meses (180 días) que ya venían siendo estables desde los 2 meses (40 días).

Como posibles efectos no deseados no se han presentado hematomas, infecciones, asimetrías (no asumibles), irregularidades permanentes o alteraciones de la cicatrización. Tan sólo cabe destacar la existencia de parestesias en la zona frontolateral transitorias, que se han recuperado espontáneamente en un máximo de 2-3 meses en 2 pacientes, aparte de las equimosis y sintomatología subjetiva transitoria habituales en las intervenciones de la zona.

\section{Discusión}

Los puntos que nos parece interesante analizar son los siguientes:

I.- Valoración del material de suspensión

Tabla I. Clasificación mallas

$\begin{array}{lll}\text { MATERIAL } & \text { BIOLOGICOS SINTETICOS } \\ & \text {-Autologos } & \\ & \text {-Aloinjertos } & \\ & \text {-Xenoinjertos }\end{array}$

ESTRUCTURA

POROSIDAD

(AMID)

ENTRAMADO

DENSIDAD

ABSORCION
MONOFILAMENTO

TIPO I

(Macroporo)

TRICOTADAS

ALTA

REABSORBIBLES

MULTIFILAMENTO

TIPO II

(Microporo)

TEJIDAS

MEDIA

IRREABSORBIBLES
TIPO III

(Macro+micro poro)

TERMOCOAGULADAS

BAJA

PARCIALMENTE REABSOBIBLES
TIPO IV

(Submicroporo) 

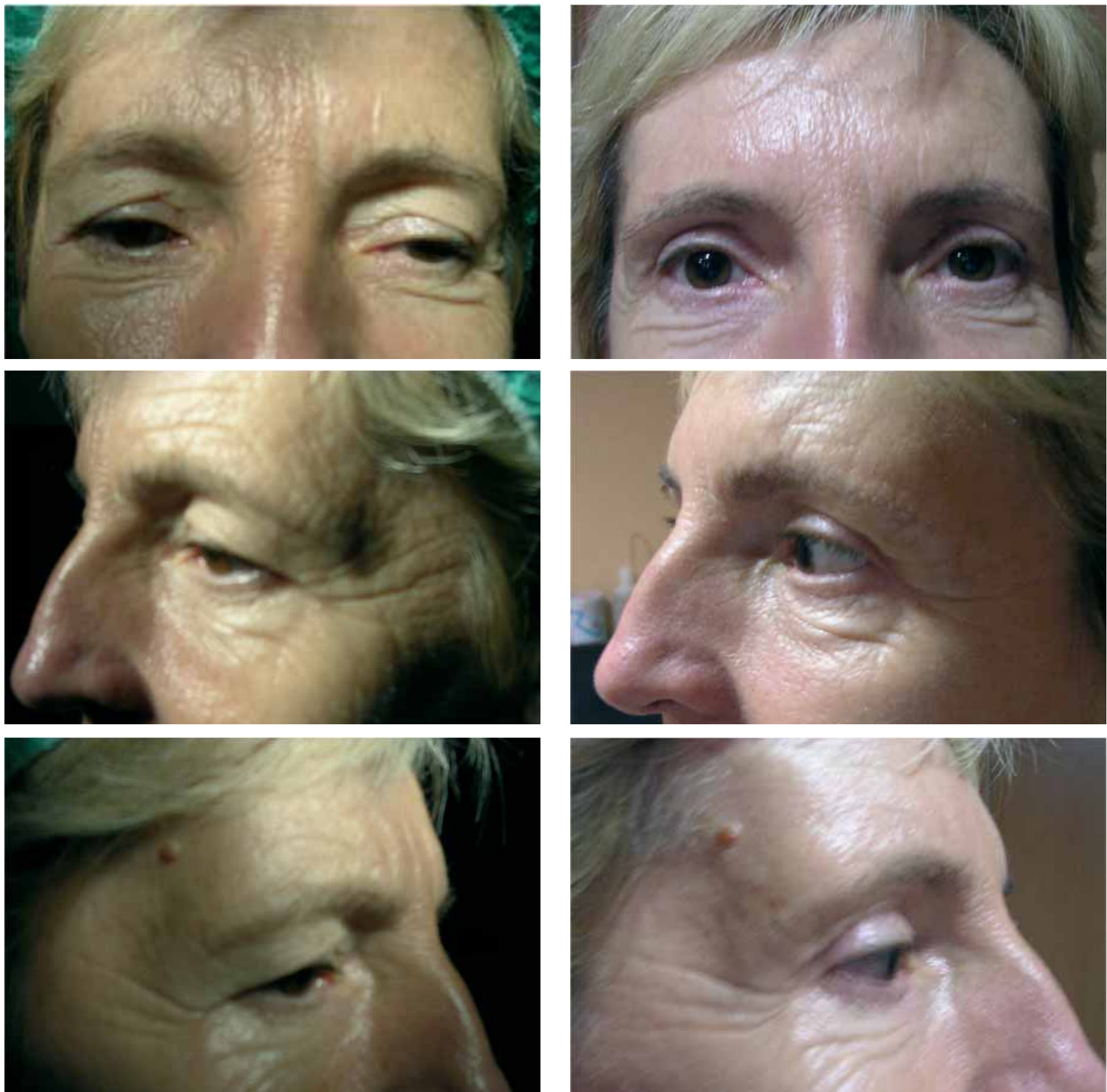

Figuras 6, 7 y 8: Imágenes pre y postoperatorias de una mujer de 45 años a la que se le ha practicado una blefaroplastia superior y nuestra técnica de lifting frontal, a los 12 meses de la intervención.

I.A.- Elección del material.

I.B.- Cualidades del material elegido

I.B.1.-Comportamiento frente a la infección

I.B.2.-Tolerancia

I.B.3.-Retracción

I.B.4.-Adhesividad

II.- Elección del Plano quirúrgico

III.- Obtención de los datos objetivos

IV.- Relación con otras técnicas

\section{I.- Valoración del material de suspensión}

\section{I.A.- Elección del material.}

En la elección del material que íbamos a utilizar para la suspensión, tuvimos en cuenta los principios que rigen para estos casos, como que no deben ser físicamente alterados por los fluidos tisulares, o que no deben producir reacción a cuerpo extraño; deben ser químicamente inertes, no carcinogénicos, no alergénicos, resistentes a las tracciones mecánicas y aptos para la esterilización sin alteración de sus cualidades (11). Las mallas de polipropileno en monofilamento cumplen en gran medida estos principios.

Existen muchos tipos de mallas y se clasifican según diferentes criterios(12) (Tabla I).

Es importante la consideración del tamaño de los poros de la malla y del grosor de los filamentos, que 

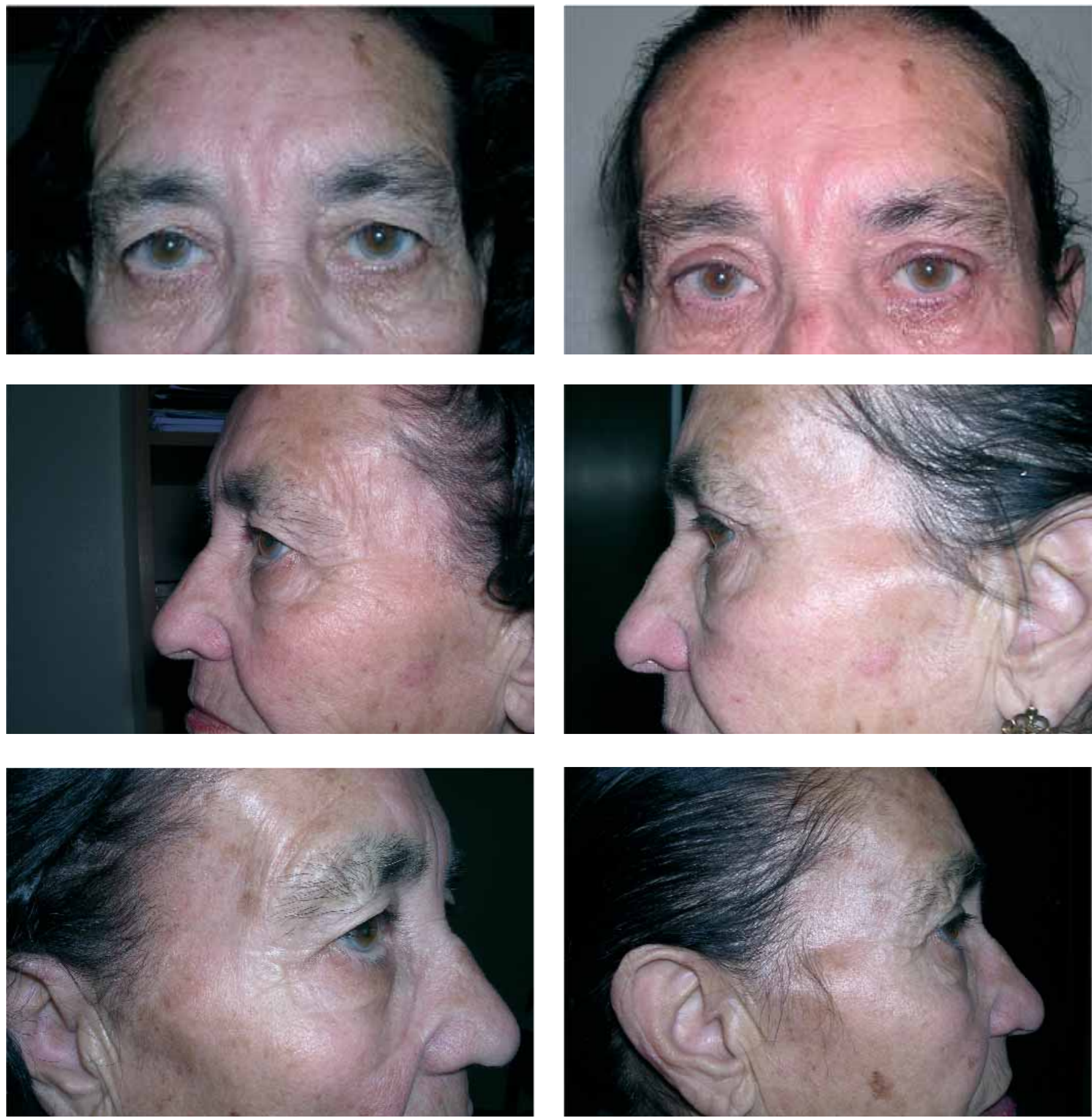

Figuras 9, 10 y 11: Imágenes pre y postoperatorias de una mujer de 72 años a la que se le practicó blefaroplastia superior y nuestra técnica de lifting frontal, sin otros refinamientos, a los 13 meses de la intervención.

definen el gramaje o densidad de la misma. Del tamaño de los poros depende en primer lugar el que la malla permita el paso a su través del tejido cicatricial. La incorporación de vasos, fibroblastos y macrófagos, y con ello la formación y aposición de fibras de colágeno, es proporcional al tamaño del poro, de modo que es necesario que tenga al menos un tamaño de 75 mm para que ésto suceda y evitar la encapsulación (11, 13-15). El grosor de la cápsula fibrosa adelgaza de forma proporcional a la cantidad de estructuras vasculares que están en contacto con la trama fibrilar
(16), que a su vez es proporcional al diámetro del poro.

\section{I.B.- Cualidades del material elegido}

\section{I.B.1.-Comportamiento frente a la infección}

La elección de la malla entre las del grupo I AMID (8) se ha basado en el comportamiento tras su inclusión en los tejidos, de tal manera que la malla monofilamentosa y con poro grande tiene mejor resistencia a la infección, ya que los leucocitos tienen un tamaño de entre 9 y $15 \mathrm{~mm}$, mientras que las bacterias solo 
cuentan con $1 \mathrm{~mm}$, refugiándose en los poros menores de $10 \mathrm{~mm}$ o en los huecos del trenzado de los multifilamentos(17). La incidencia de infección en las mallas tipo II y III AMID varía entre el 9 y el 50\% mientras que para las del tipo I ronda el 0\%(18).

\section{I.B.2.-Tolerancia}

Otra característica que hemos tenido en cuenta es la tolerancia o biocompatibildad. Esto depende, en el caso de la mallas, de dos factores: el material de fabricación y su densidad $(19,20)$. La tolerancia del material la definen tres circunstancias: la inducción de la respuesta inflamatoria, la de reacciones alérgicas y la del crecimiento tumoral. Se ha podido comprobar que cuanto menor resulta la densidad de una malla ,mejor es su tolerancia (21), con menor respuesta inflamatoria (menor número de fenómenos de apoptosis y de proliferación celular e incremento en la expresión de factor citoprotector HSP70) (22).

\section{I.B.3.-Retracción}

Una característica importante es la retracción cicatricial a que inducen debido a la proliferación de tejido fibroso-cicatricial generado por la propia malla, que lleva en todos los casos a producir una cierta retracción que se estima que será entre el 10 y el $20 \%$. Elegimos la mallas del grupo I AMID para que la integración del tejido fibroso en el entramado sea grande y la retracción no sea exagerada (19). Nosotros en realidad buscamos el que se produzca una ligera retracción que mantenga el efecto de la suspensión e incluso compense la laxitud tisular que pudiera suceder a la intervención al ceder el proceso inflamatorio postoperatorio.

El propio material, los hilos de polipropileno, se dice que estimulan la proliferación de fibroblastos productores de colágeno (23).De esta manera se renuevan los tejidos al proporcionarles esos elementos que se han ido perdiendo en el proceso del envejecimiento, tomando el tejido circundante un aspecto de mayor tonicidad e hidratación, corrigiendo por si mismo la pequeñas arrugas. Y así lo hemos observado en gran parte de los casos, efecto que en un principio no buscábamos. No hemos podido comprobar este extremo histológica o bioquímicamente; no obstante, preferimos atribuir este efecto "botulínico" al menor esfuerzo muscular del frontal como consecuencia de la propia suspensión (5). De esta opinión es también Pérez-Macías (24), que describe este efecto como consecuencia del reequilibrio de las fuerzas musculares periorbitarias depresoras y elevadoras (24). Tal vez éste podría ser el punto de partida de otro trabajo para tratar de demostrar que las suspensiones cuyo resultado es un menor esfuerzo muscular llevan a corregir las arrugas de expresión de su territorio de influencia.

De esta manera, no tenemos que retirar ningún exceso de piel para corregir la posición de la cejas ni las arrugas frontales. Este hecho ya se apuntaba en las técnicas endoscópicas, posiblemente debido a que no es el exceso de esa piel la causante de la hipotopia ciliar o de esas arrugas, ni debe ser la propia piel el punto de apoyo de la futura suspensión del sistema frontorbitario.

\section{I.B.4.-Adhesividad}

La adhesividad al tejido circundante depende de dos factores: por un lado de la estructura del entramado y de los propios filamentos que componen la malla, y por otra, de la integración del tejido fibroso en la propia malla. El tamaño del poro es también responsable de la rápida fijación de la malla por parte de la fibrina endógena(11); ésta es un característica fundamental, ya que nos basamos en ella cuando nos decidimos a ensayar esta técnica. El efecto"velcro" producido por la invasión cicatricial del entramado de la malla fijándola a los tejidos es el principio en el que nos fundamentamos para el mantenimiento a medio y largo plazo de la suspensión (12), ya que las suspensiones sustentadas en puntos de sutura o artilugios que al final se anclan puntualmente en los tejidos blandos (como los Endotine ${ }^{\circledR}$, de Coapt) terminan por perder su efecto por distensión o desgarro tisular

También del tamaño del poro depende el que las fibras de colágeno se dispongan en formación orientada a los filamentos de la malla, que es responsable de las cualidades de resistencia y elasticidad del conjunto $(11,25)$.

\section{II.- Elección del Plano quirúrgico}

Esto nos lleva a la consideración de la segunda decisión: el plano de colocación.

Los métodos de suspensión quirúrgica, bien sea abierta o endoscópica suelen elegir como plano de despegamiento el plano subperióstico, que es normalmente amplio, para conseguir que el aparato músculoaponeurótico suprayacente le auxilie en su efecto lifting y además evitar así la lesión de los filetes nerviosos que cruzan la zona.

Estas técnicas son de mayor complejidad técnica, y exigen un instrumental caro y sofisticado $(10,26)$. Al comparar los resultados no vemos sin embargo diferencias. Si bien con nuestra técnica no llegamos a poder tratar por esta vía los efectos de los músculos depresores mediales (corrugador y prócerus), en caso necesario se puede complementar seccionándolos desde el extremo interno del abordaje de la blefaroplastia, o mediante un sencillo gesto como es la ino- 
culación en los mismos con Toxina Botulínica tipo A, lo que consigue completar el resultado manteniendo la sencillez técnica.

Los métodos de suspensión basados en la tracción cutánea con hilos arponados se ven obligados a permanecer en el plano subcutáneo para suspender el tejido y aprovechar el efecto generador de colágeno del propio material.

Para nuestra técnica hemos elegido el plano supraperióstico ya descrito en 1992 por De la Plaza para lifting frontal (27), por necesitar de un espacio bien vascularizado que favorezca la proliferación fibrosa rápida, de manera que la adhesividad de la malla sea rápida e intensa, y al tiempo poder fijarla a un plano más bien rígido como es la aponeurosis temporal superficial cuya relación con la piel suprayacente es estrecha, aprovechando además, en cierta medida el efecto productor de colágeno que en realidad nos lo hemos encontrado accidentalmente. Además, es el menos traumático de todos los planos posibles.

\section{III.- Obtención de los datos objetivos}

Aunque otros autores han propuesto otras alternativas de medición de los resultados(10), nos parece más exacto el nuestro, ya que los puntos de referencia residen en el marco óseo y no en partes blandas que están sujetas a variaciones ambientales.

\section{IV.- Relación con otras técnicas}

Bessins (28) en la descripción de su técnica R.A.R.E. (Reverse and Repositioning Effect) propone una división facial vertical opuesta a la horizontal clásica, considerando que en la zona medial (Procara) y lateral (Metacara) de la cara la piel tiene bastante fijación, en el primer caso a estructuras óseas y en el segundo por estructuras ligamentosas (ligamentos de Furnas), existiendo entre ellas una zona (Mesocara) que incluiría la porción vertical que desde el área temporal con la cola de la ceja continua hacia el canto ocular externo, pómulo, mejilla y tercio externo de los labios hasta llegar a la barbilla; es la única zona expuesta a la acción gravitacional que sigue a los procesos tisulares que se producen con el paso del tiempo, según hemos considerado anteriormente.

Según este autor, para reposicionar los tejidos, sólo habría que actuar sobre esta zona evitando dañar las zonas vecinas que de por si no sufren alteraciones. La suspensión en su técnica la realiza con hilos o con elementos de fijación osteo-galear como los Endotine ${ }^{\circledR}$, (de Coapt).

En el caso de que sus premisas fueran ciertas creemos que tendrían mejor resultado, y a mas largo plazo, si la suspensión se realizara con una malla, basándonos en sus características de adhesividad, que descargaría el peso de la suspensión en una mayor superficie y no dejaría el peso de esa responsabilidad a unos pocos puntos tisulares blandos aislados.

\section{Conclusiones}

Creemos que la técnica que presentamos es una buena alternativa a las técnicas de suspensión y ritidectomía ya mencionadas: en el caso de las quirúrgicas por su simplicidad técnica e instrumental y escaso traumatismo; en el caso de las de los hilos arponados porque la tracción en nuestro caso se hace de una manera más difusa, sin crear efecto brida; y en los dos casos, por sumar los efectos de unos y otros, garantizar unos resultados duraderos, mínimas secuelas cicatriciales y sobre todo por su bajo coste económico.

\section{Agradecimientos}

Agradecemos a D ${ }^{a}$ Elina Vilar Beltrán los trabajos de traducción, sobre todo en Inglés, que han sido fundamentales para la consecución de este trabajo.

\section{Dirección del autor}

\author{
Dr. Juan P. Aracil Kessler \\ UCSI \\ Hospital Provincial de Castellón \\ Avda. Dr. Clará 19 \\ 12002 Castellón. España
}

\section{Bibliografía}

1. Hinderer U.T. "La Cirugía Plástica- Estética. Psicoterapia quirúrgica”. Cir. Plast. Iberlatinoamer. 1990, 16: 15.

2. García Velasco J., García Velasco M., Apellaniz Campo A., Guerrero Pérez R., García Casas S. "Blefaroplastia superior extendida”. Cir. Plast. Iberlatinoamer. 1998, 24 (2) : 119.

3. Lott Caldeira A.M., Lucas A., Grigalek G. "Envejecimiento Facial. Papel de la órbita senil”. Cir. Plast. Iberlatinoamer. 1996, 22 (1) :21.

4. Knize D.M. "An anatomically based study of the mechanism of eyebrow ptosis”. Plast. Reconstr. Surg. 1996, 97: 1321.

5. Trepsat F., Morax S. "Chirugie esthetique fronto-orbito-palpébrale". Encycl.Méd.Chir.(Editions Scientifiques et Médicales Elsevier SAS Paris) Technique chirurgicales - Chirugíe plastique, 1996, Pp 45-650.

6. Pollock R.A., Gossman D. "Avoiding the incomplete blepharoplasty: refinements in aesthetic upper eyelid surgery through adjuntive procedures". Perspect. In Plast. Surg. 1992, $6: 39$.

7. Tessier P. "The liftinf facial sous-periosté". Ann. Chir. Plast.Esthét. 1989, 34: 193.

8. Amid P.K., Shulman A.G., Lichtenstein I.L.,Hakakha M. "Biomaterials for abdominal wall hernia surgery and principles of their applications". Langenbecks Arch.Chir. 1994; 379 (3): 168.

9. Amid P.K. "Classification of biomaterials and their related complications in abdominal wall hernia surgery". Hernia 1997, 1: 5.

10. De la Fuente A., Santamaría Robredo A.B., Díaz Infante J.L. "Lifting frontal Endoscópico". Cir. Plast. Iberlatinoamer. 1995 , 21 (3): 267.

11. Greca F.H., de Paula J.B., Biondo-Simoes M.L.P., da Costa F.D., 
da Silva A.P.G., Tima S., Mansur A. "The influence of differing pore sizes on the biocompability of two polyprpylene meshes in the repair of abdominal defects". Hernia 2001, 5:59.

12. Galmés Belmonte,I.; Diaz Gómez, E. “¿Son iguales todos los sistemas empleados para corregir la incontinencia urinaria mediante mallas libres de tensión?" Actas Urológicas Españolas Jul/Ago 2004 pags: 487

13. Adloff M., Arnaud J.P. "Etude expérimentale de la résistance et de la tolérance biologique des matériaux prothétiques utilices dans la réparation des pertes de substance de la paroi abdominale". Chirugie 1976, 102: 390.

14. Amid P.K., Shulman A.G., Lichtenstein I.L., Hakakha M. "Biomaterials for "tension-free" hernioplasties and principles of their applications". Minerva Chir. 1995, 50: 821.

15. Merrit K., Shafer J.W., Brown S.A. "Implant-site infection rates with porous and dense material". Biomed. Mater. Res. 1979, 13: 101.

16. Sanders J.E., Stiles C.E., Hayes C.L. "Tissue Response to singlepolymer fibers of varying diameters: Evaluation of fibrous encapsulation and macrophage density". J. Biomed. Mater. Res. 2000, 52 (1): 231 .

17. Kingle U., Junge K., Spellerberg B.,Piroth C., Klosterhalfen B, Schumpelick V. "Do multifilament alloplastic meshes increase the infection rate?. Analysis of polymeric surface, the bacteria adherence and the vivo consequences in a rat model". J.Biomed.Mater.Res. 200263 (6) 765.

18. Cervigny M., Natale F. "The use of synthetics in the treatment of pelvic organ prolapse". Curr. Opin.Urol. 2001, 11(4): 429.

19. Debodinance P.,Delporte P., Engrand J.B.,Boulogne M. "Development of better tolerated prostetic materials: applications in gynecological surgery". J.Gynecol.Obstet.Biol.Reprod. (Paris) 2202, 31 (6):527.
20. Falconer C., Soderberg M.,Blomgren B., Ulmsten U. "Influence of different sling materials on connective tissue metabolism in stress urinary incontinent woman". Int.Urogynecol.J.Pelvic FloorDysfunct. 200112 Suppl. (2) : S19.

21. Kingle U., Klosterhalfen B.,Conze J., Limberg W., Obolenski B., Ottinger A.P., Schumpelick V. "Modified Mesh for hernia repair that is adapted to the physiology of the abdominal wall". Eur. J. Surg. 1998 Dec ; 164 (12): 951.

22. Junge K., Kingle U., Rosh R., Klosterhafen B., Schumpelick V. "Functional and morphologic properties of a modified mesh for inguinal hernia repair". World J. Surg. 2002; 26 (12): 1472.

23. Viacheslavovna Taran N. "Armado de la piel de la cara y cuello con hilos quirúrgicos especiales". Tesis doctoral (Traducción al castellano). Academia de Ciencias Medicas de Rusia. Instituto de Cirugía A.V. Vishnevski. Moscú 1999, p80.

24. Pérez Macías Macías J.M. "Divergentes o convergentes puntos de vista en el rejuvenecimiento del tercio superior de la cara". Cir. Plast. Iberlatinoamer. 2002, 28 (2): 95.

25. Davila J.C., Lautsch E.V., Palmer T.E. "Some Physical factors affecting the acceptance of synthetic materials as tissue implants". Ann. NY Acad. Sci. 1968, 146: 138.

26. Serra Renom J.M., Vila Rovira R. "Endoscopia en Cirugía Plástica y Estética". Ed. Masson Barcelona 1.995 , P136.

27. De la Plaza R., Valiente E., Arroyo J.M. "Supraperiosteal Lifting of the upper two-thirds of the face". Itern Workshop on facial rejuvenation. Baltimore 1992

28. Bessins T. "The R.A.R.E. technique (Reverse and Repositioning Effect); the renaissance of the Aging Face and Neck". Aesth. Plast. Surg. 2004, 28: 127

\section{Comentario al trabajo ulifting frontal apoyado en malla de polipropilenon}

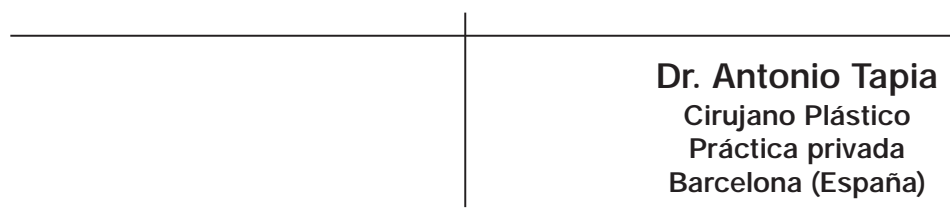

Múltiples trabajos se han realizado en la búsqueda de un tratamiento que corrija la ptosis de tercio medio y externo de la ceja ya que se presenta en personas jóvenes y de mediana edad dando lugar a una imagen de cansancio, tristeza o envejecimiento precoz. Este factor edad es el que limita muchas veces la amplitud del tratamiento quirúrgico y se limita a técnicas endoscópicas o directas de despegamiento en un plano subperióstico (liberando éste 1 ó $2 \mathrm{~cm}$. antes de la ceja) o preperióstico y fijación de la aponeurosis temporal superficial a tejido perióstico y aponeurótico u osteogalear.

Aracil Kessler y colaboradores presentan una técnica que se centra, no en la vía de abordaje, común con otras técnicas de suspensión, sino en el estudio, solución y aplicación de una malla de polipropileno Hermesh 5 flat mesh que sirva de elemento de suspensión y creación de una fibrosis que sirva para adherir el plano preperióstico.

Felicito al Dr. Aracil Kessler y a sus colaboradores por el estudio exhaustivo realizado para seleccionar el material y su buena tolerancia; pero es difícil valorar los resultados por los ejemplos mostrados en las figuras $6,7,8,9,10$ y 11. Primero: no creo que sea el paciente indicado para este tipo de cirugía por presentar un envejecimiento general. Segundo: ¿A qué se debe la mejora? ¿A la simple blefaroplastia superior?, al edema y fibrosis que se crea en el despegamiento ¿la liberación de grasa palpebral, retrociliar y del ligamento orbitario? que su liberación es lo que realmente moviliza los tejidos frontales (Knize).

Lo importante es que la malla aumenta la fibrosis y por lo tanto hace más duradero el resultado. El seguimiento de más tiempo en el resultado, la oportuna selección del paciente, a ser posible más joven y una fotografía más demostrativa del resultado (es lo que se ve el paciente) abren una vía más amplia de investigación en este interesante trabajo no sólo por el material recogido sino por el mayor o menor efecto de suspensión-tracción. 